\title{
REPETITION THRESHOLDS FOR SUBDIVIDED GRAPHS AND TREES
}

\author{
${\text { Pascal } \text { OChem }^{1} \text { And Elise VAslet }}^{2}$
}

\begin{abstract}
The repetition threshold introduced by Dejean and Brandenburg is the smallest real number $\alpha$ such that there exists an infinite word over a $k$-letter alphabet that avoids $\beta$-powers for all $\beta>\alpha$. We extend this notion to colored graphs and obtain the value of the repetition thresholds of trees and "large enough" subdivisions of graphs for every alphabet size.
\end{abstract}

Mathematics Subject Classification. 68R15.

\section{INTRODUCTION}

A non-repetitive coloring $f$ of a graph is a vertex coloring containing no square, that is, the graph does not contain a non-intersecting path $v_{1}, \ldots, v_{2 r}$ such that $f\left(v_{i}\right)=f\left(v_{i+r}\right)$ for all $i \in[1, r]$.

This notion can be extended by considering repetitions of fractional exponent, as it has been done in the framework of combinatorics on words.

Up to now, the most studied problem is the following: we fix the exponent (i.e., exponent 2, corresponding to squares) to be avoided and minimize the number of colors, i.e., the alphabet size. For large subdivisions of graphs, this so-called nonrepetitive chromatic number is proved to be 3 [6]. The non-repetitive chromatic number of trees is 4 [4].

Aberkane and Currie [1] considered the problem the other way. They fix the alphabet size and study what exponents can be avoided. They show that there

\footnotetext{
Keywords and phrases. Combinatorics on words, repetition threshold, square-free coloring.

1 CNRS, LRI, Université Paris-Sud 11, 91405 Orsay Cedex, France. ochem@lri.fr

2 IML, UMR 6206, Université Aix-Marseille II, Campus de Luminy, Case 907, 13288 Marseille

Cedex 9, France. vaslet@iml.univ-mrs.fr
} 
exist binary circular words of every length avoiding exponents strictly greater than $5 / 2$. The cycle of length 5 shows that this is the best possible bound.

A notion of repetition threshold (similar to Dejean's repetition threshold [3]) can then be defined for graphs. Aberkane and Currie's result sets it to $5 / 2$ for cycles.

We settle the problem of finding the repetition thresholds of two graph classes, namely the subdivided graphs and the trees, for every alphabet size $k$. This improves earlier results on nonrepetitive coloring on these classes $[4,6]$.

\section{Preliminaries}

Let $k \in \mathbb{N}$ and let $f$ be a $k$-coloring of a finite graph $G$. We call factor the sequence of colors on a non-intersecting path in a $k$-colored graph $G$. We recall the usual notions of period and exponent: a word $w$ is a repetition with period $p$ and excess $e$ if $w=p e$, and $e$ is a prefix of $w$. The exponent of the repetition $w$ is the ratio $\exp (w)=\frac{|p e|}{|p|}$. A word or a colored graph is said to be $\left(\alpha^{+}, n\right)$-free if it does not contain as a factor a repetition with exponent strictly greater than $\alpha$ and period of length at least $n$. We use $\alpha^{+}$-free as a short notation for $\left(\alpha^{+}, 1\right)$-free. Let $G$ be a graph. We define the repetition threshold $R T(k, G)$ by

$$
\inf _{k \text {-coloring } f} \sup \{\exp (w) \mid w \text { is a factor in the } k \text {-coloring } f \text { of } G\} \text {. }
$$

For a graph class $\mathcal{G}$, we define $R T(k, \mathcal{G})=\sup _{G \in \mathcal{G}} R T(k, G)$. Let us express some known results with this definition: $R T(2, \mathcal{C})=5 / 2[1]$ and $R T(4, \mathcal{T}) \leq 2[4]$, where $\mathcal{C}$ the class of cycles and by $\mathcal{T}$ the class of trees. A subdivision of a graph $G$ is a graph obtained from $G$ by a sequence of edge subdivisions. A subdivision of an edge $\{u, v\}$ consists in the addition of a new vertex $w$ and the replacement of the edge $\{u, v\}$ by the edges $\{u, w\}$ and $\{w, v\}$. It has been shown that for every graph $G$, there exists a subdivision $G_{s}$ of $G$ such that $R T\left(3, G_{s}\right)<2[6]$.

The Thue-Morse word $w_{T M}=011010011001011010010110011010 \ldots$ is the fixed point of $0 \rightarrow 01,1 \rightarrow 10$.

Let $w[i, \ldots, j]$ denote the subword $w_{i} w_{i+1} \ldots w_{j}$ of the word $w$.

Finally, we denote by $\Sigma_{k}$ the $k$-letter alphabet $\{0,1, \ldots, k-1\}$.

\section{REPETITION THRESHOLD FOR SUBDIVIDED GRAPHS}

For a given $k$, we define the real number

$$
\alpha_{k}=\sup _{G} \inf _{G_{s} \text { subdivision of } G} R T\left(k, G_{s}\right) .
$$

By abuse of notation, we set $R T(k, \mathcal{S})=\alpha_{k}$. We can see $\mathcal{S}$ as the pseudo-class of "large enough" subdivisions of graphs. 


\section{Theorem 3.1.}

- $R T(2, \mathcal{S})=\frac{7}{3}$,

- $R T(3, \mathcal{S})=\frac{7}{4}$

- $R T(k, \mathcal{S})=\frac{3}{2}$, for $k \geq 4$.

Proof. We first prove the upper bounds. For any graph $G$, we construct a subdivision $G_{s}$ of $G$ and a suitable coloring of $G_{s}$. Without loss of generality, we can suppose that $G$ is a (large) complete graph. Let $e$ denote the number of edges of $G$. For each $k$, we consider a set $W_{k}$ containing $2 e$ words over the $k$-letter alphabet such that all words in $W_{k}$ have a non-empty common prefix and a non-empty common suffix. An edge in $G$ is replaced by a chain of vertices of degree 2 in $G_{s}$. A vertex of degree at least 3 in $G_{s}$ thus corresponds to an original vertex in $G$ and is said big. Such a chain consists of two half-chains. We color each half-chain with a distinct word $w \in W_{k}$ such that the first letter of $w$ corresponds to the color of a big vertex. The last vertex of an half-chain is identified with the last vertex of the other half-chain of a same chain. This common vertex is called the center of the chain and is colored with the common last letter of all words in $W_{k}$. A vertex is said to be special if it is a big vertex or a center. Two half chains sharing a special vertex are said to be opposite since they have opposite reading directions.

A morphism $h: \Sigma_{s}^{*} \rightarrow \Sigma_{e}^{*}$ is synchronizing if for any $a, b, c \in \Sigma_{s}$ and $v, w \in \Sigma_{e}^{*}$, if $h(a b)=v h(c) w$, then either $v=\varepsilon$ and $a=c$, or $w=\varepsilon$ and $b=c$. In order to prove upper bounds on the exponent of repetitions, it is convenient to use synchronizing uniform morphisms together with the following lemma from [5]:

Lemma 3.2. Let $\alpha, \beta \in \mathbb{Q}, 1<\alpha<\beta<2$ and $n \in \mathbb{N}^{*}$. Let $h: \Sigma_{s}^{*} \rightarrow \Sigma_{e}^{*}$ be $a$ synchronizing q-uniform morphism (with $q \geq 1$ ). If $h(w)$ is $\left(\beta^{+}, n\right)$-free for every $\alpha^{+}$-free word $w$ such that $|w|<\max \left(\frac{2 \beta}{\beta-\alpha}, \frac{2(q-1)(2 \beta-1)}{q(\beta-1)}\right)$, then $h(t)$ is $\left(\beta^{+}, n\right)$-free for every (finite or infinite) $\alpha^{+}$-free word $t$.

Case $k=2$ :

We consider a set $W_{2}$ of (distinct) factors of the Thue-Morse word with prefix 0110010 and suffix 1011001. Suppose that there exists a repetition $r$ in $G_{s}$ with exponent strictly greater than $\frac{7}{3}$. If $r$ contains at most one big vertex (resp. at most one center), then either:

- $r$ is a factor of $w_{T M}$ and its exponent is at most 2 .

- $r$ has period at most 6 and we check that its exponent is at most $\frac{7}{3}$.

- The period of $r$ contains exactly one occurrence of at least one factor in $\{01001101,10110010\}$. Those factors appear near the big vertex or the center because of the prefix 0110010 and the suffix 1011001 in the half chains, and since they are not factors of $w_{T M}$, we have a contradiction.

If $r$ contains at least two big vertices and at least two centers, then two half-chains should be matched in $r$. This is a contradiction because half-chains are distinct. 
Case $k=3$ :

We consider the synchronizing 132-uniform morphism $h$ in [5] such that for any $\frac{5}{4}^{+}$-free word $t \in \Sigma_{6}^{*}, h(t) \in \Sigma_{4}^{*}$ is $\frac{7}{5}^{+}$-free. From [2], there exists an infinite $\frac{5}{4}^{+}$-free word $w \in \Sigma_{5}^{*}$ such that one of the letter, say 4 , appears every other six positions. For a fixed integer $i \geq 1$, we define the infinite word $w_{\langle i\rangle} \in \Sigma_{6}^{*}$ obtained from $w$ by replacing every other $i$ occurrences of the letter 4 with the letter 5 , so that the frequency of the letter 5 in $w_{\langle i\rangle}$ is $\frac{1}{6 i}$. We consider a set $S$ of $2 e$ words of length $c$ with prefix 0123 and suffix 1230, such that the $i^{\text {th }}$ element in $S$ is a factor of $h\left(w_{\langle i\rangle}\right)$, for $1 \leq i \leq 2 e$. Notice that both 0123 and 1230 appear in every factor of length 100 of the $h$-image of any word. Words in $S$ are in particular $\left(\frac{7}{5}^{+}\right)$-free words over $\Sigma_{4}$.

Consider also the following synchronizing 48-uniform morphism $m$ :

$0 \mapsto 012010201202120121020102120121012010201210212021$

$1 \mapsto 012010201202120121012021020102120121012010212021$

$2 \mapsto 012010201202101210212012101202120102012021020121$

$3 \mapsto 012010201202101210201202120121012010212021020121$

The set $W_{3}$ contains the words of the form $w=m(x) 01201020120$, with $x \in S$. So the words in $W_{3}$ have length $d=48 c+11$. Since the morphisms $h$ and $m$ are synchronizing, we can take $d$ large enough to ensure that the factors of length $d / 100$ of distinct words in $W_{3}$ do not contain the same number of factors $m(h(5))$, and are thus distinct. The prefixes and suffixes of length 11 and the factors of length 52 of words in $W_{3}$ contain the factor 01201, whereas words in $W_{3}$ do not contain the mirror factor 10210. Using Lemma 3.2, we can check that the words in $W_{3}$ are $\left(\frac{7}{4}^{+}\right)$-free and $\left(\frac{3}{2}^{+}, 14\right)$-free.

Suppose that there exists a repetition $r=u v u$ in $G_{s}$ with exponent strictly greater than $\frac{7}{4}$, that is, $|u|>3|v|$. Obviously, $r$ cannot be contained in one half-chain. A computer check shows that the period $p=|u v|$ of $r$ must be at least 68 , by looking at the neighborhood of a special vertex. We first consider the case where $r$ is contained in two (opposite) half-chains. If the common special vertex belongs to $v$, then $|u v| \geq 68$ and $|u|>3|v|$ gives $|u| \geq 52$. This is a contradiction since the factor 01201 appearing in one occurrence of $u$ would induce a forbidden factor 10210 in the word in $W_{3}$ corresponding to the opposite chain containing the other ocurrence of $u$. If the common special vertex belongs to $u$, then the restriction of $r$ to one of the half-chain is a repetition of period $p \geq 68$ and exponent at most $\frac{3}{2}$. To complete it and obtain the repetition $r$, there must exist a factor $u^{\prime}$ of $u$ with size $\left|u^{\prime}\right|>p\left(\frac{7}{4}-\frac{3}{2}\right) \geq 68 \times \frac{1}{4}>11$. So this occurrence of $u^{\prime}$ contains the factor 01201 that is near the special vertex. Since the matching occurrence of $u^{\prime}$ is contained in the opposite half-chain, we have again a contradiction with the factors 01201 and 10210. Suppose now that $r$ contains at least two special vertices. Since words in $W_{3}$ are $\left(\frac{3}{2}^{+}, 14\right)$-free, we have $\frac{3}{2}|u v| \geq d$. Recall that $|u|>3|v|$, which gives $|u|>\frac{d}{2}$ and $|r|>\frac{7}{6} d$. Then, there exists a factor $u^{\prime}$ of $u$ such that 
either $\left|u^{\prime}\right| \geq 52$ and both occurrences of $u^{\prime}$ are in half-chains with opposite reading directions, or $\left|u^{\prime}\right| \geq d / 100$ and both occurrences of $u^{\prime}$ are in distinct half-chains with the same reading direction. In both cases, we have a contradiction.

Case $k=4$ :

We consider the set $S$ defined in the case $k=3$ and the following 11-uniform synchronizing morphism $m$ :

$0 \mapsto 01320213032$

$1 \mapsto 01312023203$

$2 \mapsto 01232120323$

$3 \mapsto 01213231302$

The set $W_{4}$ contains the words of the form $w=m(x) 01$, with $x \in S$. As in the case $k=3$, we can suppose that the factors of length $d / 100$ of distinct words in $W_{4}$ are distinct, where $d=11 c+2$ is the length of words in $D$. The prefixes and suffixes of length 2 and the factors of length 12 of words in $W_{4}$ contain the factor 01, whereas words in $W_{4}$ do not contain the mirror factor 10. Using Lemma 3.2, we can check that the words in $W_{4}$ are $\left(\frac{3}{2}^{+}\right)$-free and $\left(\frac{10}{7}^{+}, 34\right)$-free.

Suppose that there exists a repetition $r=u v u$ in $G_{s}$ with exponent strictly greater than $\frac{3}{2}$, that is, $|u|>|v|$. Obviously, $r$ cannot be contained in an half-chain We check that the period $p=|u v|$ of $r$ must be at least 22, by looking at the neighborhood of a special vertex. We first consider the case where $r$ is contained in two (opposite) half-chains. If the common special vertex belongs to $v$, then $|u v| \geq 22$ and $|u|>|v|$ gives $|u| \geq 12$. This is a contradiction since the factor 01 appearing in one occurrence of $u$ would induce a forbidden factor 10 in the word in $W_{4}$ corresponding to the opposite chain containing the other ocurrence of $u$. If the common special vertex belongs to $u$, then $u$ must contain a factor 01 or 10 that cannot be matched in the other occurrence of $u$. Suppose now that $r$ contains at least two special vertices. Since words in $W_{4}$ are $\left(\frac{10}{7}^{+}, 34\right)$-free, we have $\frac{10}{7}|u v| \geq d$. Recall that $|u|>|v|$, which gives $|u|>\frac{7}{20} d$ and $|r|>\frac{21}{20} d$. Then, there exists a factor $u^{\prime}$ of $u$ such that either $\left|u^{\prime}\right| \geq 12$ and both occurrences of $u^{\prime}$ are in half-chains with opposite reading directions, or $\left|u^{\prime}\right| \geq d / 100$ and both occurrences of $u^{\prime}$ are in distinct half-chains with the same reading direction. In both cases, we have a contradiction.

Now, we prove the lower bounds.

Let us call spider a tree with at most one vertex of degree strictly greater than two. Notice that every subdivision $G_{s}$ of a spider $G$ contains $G$ as a subgraph. For each $k$, we give a spider $G$ such that any $k$-coloring of $G$ contains a repetition of exponent at least the value of $R T(k, \mathcal{S})$ given in the theorem. Every subdivision $G_{s}$ of this spider $G$ contains $G$ as a subgraph and thus a repetition of exponent at least $R T(k, \mathcal{S})$, which proves the lower bound. 
Case $k=2$ :

Every $\left(\frac{7}{3}^{+}\right)$-free binary word of length 4 has a prefix in $F=\{00,11,010,101$, $0110,1001\}$. Consider the spider $G$ on 13 vertices containing a vertex $v$ of degree 4 incident to 4 paths of length 3 . Without loss of generality, $v$ gets color 0 . Since there are 4 paths to color in $G$ and only 3 words in $F$ starting with 0 , two paths are colored with the same word in $F$. Now, for any factor $f[1, d]$ in $F$, the factor $f_{d} f_{d-1} \ldots f_{2} f_{1} f_{2} \ldots f_{d-1} f_{d}$ is a repetition of exponent at least $\frac{7}{3}$.

Case $k=3$ :

Dejean [3] proved that the repetition threshold of a path on 39 vertices is $\frac{7}{4}$. Such a path is a spider.

Case $k \geq 4$ :

We consider the spider $G=K_{1, k}$, that is, $G$ contains a vertex $v$ with degree $k$ and its neighbors. We have to color $k+1$ vertices, $v$ and its neighbors, using $k$ colors only, so at least two of them get the same color. If $v$ has the same color as one of its neighbors then we get a square, and if two neighbors of $v$ have the same color then we get a repetition of exponent $\frac{3}{2}$.

\section{REPETITION THRESHOLD FOR TREES}

\section{Theorem 4.1.}

- $R T(2, \mathcal{T})=\frac{7}{2}$

- $R T(3, \mathcal{T})=3$,

- $R T(k, \mathcal{T})=\frac{3}{2}$, for $k \geq 4$.

Proof. We define the family $U_{n}, n \geq 0$ of rooted trees as follows: $U_{0}$ is a vertex, $U_{n+1}$ is obtained from $k^{n}+1$ copies of $U_{n}$ by adding a root vertex adjacent to the root of each copy. The family $U_{n}$ is universal, that is, every (rooted) tree is the subgraph of $U_{n}$ for some $n$. We thus have $R T(k, \mathcal{T})=\lim _{n \rightarrow \infty} R T\left(k, U_{n}\right)$. A tree is level-colored if it is colored such that all vertices at the same depth have the same color.

We prove now that in any $k$-coloring of $U_{n}$, there exists in $U_{n}$ a level-colored complete binary subtree of height $n$. Suppose that the property holds for $U_{n}$ and consider any coloring of $U_{n+1}$. We have $k^{n}+1$ copies of $U_{n}$, each containing a levelcolored binary tree, and there are at most $k^{n}$ ways to color one of these binary trees. So two level-colored binary trees have the same coloring, and this creates the expected level-colored binary tree in $U_{n+1}$.

The repetition threshold of a $k$-coloring of $U_{n}$ is at least the largest exponent of a repetition in a level-colored complete binary tree of height $n$ that it contains. Moreover, the largest exponent of a repetition in a level-coloring is the same for $U_{n}$ and for a binary complete tree of height $n$, because the set of factors is the same in these two trees. 
The study of $R T(k, \mathcal{T})$ is thus reduced to that of level-colorings of complete binary trees, so we can consider the word $w=w_{1} w_{2} \ldots$ such that $w_{i}$ is the color appearing at depth $i-1$. The factors of the level-colored complete binary tree are exactly those of the form $w_{m+l} w_{m+l-1} \ldots w_{m+1} w_{m} w_{m+1} \ldots w_{m+r-1} w_{m+r}$.

We first prove the upper bounds. For each $k$, we consider an infinite word $w$ over the $k$-letter alphabet such that for all $l, r$, and $m$, the exponent of $w_{m+l} w_{m+l-1} \ldots w_{m+1} w_{m} w_{m+1} \ldots w_{m+r-1} w_{m+r}$ is at most the expected value of $R T(k, \mathcal{T})$. Without loss of generality, we suppose that $l \leq r$.

Case $k=2$ :

We choose $w$ to be the Thue-Morse word $w_{T M}$. Notice that the factor $w_{m+2} w_{m+1}$ $w_{m} w_{m+1} w_{m+2}$ is in $\{10001,01010,11011,00100,10101,01110\}$, so it is not a factor of $w_{T M}$. Suppose that $w_{m+l} w_{m+l-1} \ldots w_{m+1} w_{m} w_{m+1} \ldots w_{m+r-1} w_{m+r}$ is a repetition with a period of length $p \geq 2$ and exponent strictly greater than $\frac{7}{2}$ (a repetition with period of length 1 has exponent at most 3 ). If $l=1$, then the exponent is $2+\frac{1}{p} \leq \frac{5}{2}<\frac{7}{2}$. If $l \geq 2$, then the factor $w_{m+2} w_{m+1} w_{m} w_{m+1} w_{m+2}$ must appear in $w[m, m+r]$, which is a factor of $w_{T M}$. A contradiction.

Case $k=3$ :

We choose $w$ to be the image of any ternary square-free word by the morphism $0 \mapsto 00,1 \mapsto 11,2 \mapsto 22$ which doubles every letter. The tree thus contains the cubes 000,111 , and 222 , but no cube of period at least two. To see this, consider a potential cube uuu of period at least two as two overlapping occurrences of the square $u u$. Since $w$ contains no square of period at least two, the leftmost occurrence of $u u$ cannot be contained in the left branch of the cube factor. So the leftmost factor $u u$ contains the factor $b a b$ where $a, b \in \Sigma_{3}$ and $a$ is the color of the root. This is a contradiction, since the matched occurrence of $b a b$ in the rightmost factor $u u$ is contained in the right branch of $u u u$ but bab is not a factor of $w$.

Case $k=4$ :

We choose $w$ to be any $\left(\frac{3}{2}\right)$-free word over $\Sigma_{4}$. Notice that $w$ has no factors of the form $a a$ nor $a b a$ where $a$ and $b$ are letters. So $w$ contains no palindrome of size at least two. Suppose that $u v u$ is a repetition of exponent strictly greater than $\frac{3}{2}$, that is, $|u|>|v|$, and suppose w.l.o.g that 01 is a prefix of $u$. The first factor $u$ cannot contain a vertex and two of its sons, since this creates a factor $a b a$ that cannot be matched in the other factor $u$. So the first occurrence of $u$ is a prefix of the left branch $w_{m+l} w_{m+l-1} \ldots w_{m+1} w_{m}$.

Without loss of generality, $w_{m}$ is the last letter of $u$, since otherwise the factor $w_{m+l} w_{m+l-1} \ldots w_{m+2} w_{m+1} w_{m+2} \ldots w_{m+r-1} w_{m+r}$ rooted at level $m+1$ is the repetition $u v^{\prime} u$ where $v^{\prime}$ is the suffix of $v$ of length $|v|-2$, so that the exponent of $u v^{\prime} u$ is greater than the exponent of $u v u$. Thus $|u|=l+1$, and since $|v|<|u|$, we can consider the following cases on the right branch:

- $|v|=l$ : the factor $w_{m+l} w_{m+l+1}$ should be 00 , a contradiction.

- $|v|=l-1$ : the factor $w_{m+l-1} w_{m+l} w_{m+l+1}$ should be 101 , a contradiction. 
- $|v|=l-t, t \geq 2$ : the factor $w_{m+l-t+1} \ldots w_{m+l}$ should be a palindrome of size at least two, a contradiction.

Now, we prove the lower bounds.

For each $k$, we prove that for any infinite word $w$ over the $k$-letter alphabet, there exist $l, r$ and $m$, such that the exponent of $w_{m+l} w_{m+l-1} \ldots$ $w_{m+1} w_{m} w_{m+1} \ldots w_{m+r-1} w_{m+r}$ is at least $R T(k, \mathcal{T})$.

Case $k=2$ :

It is easy to check that every long enough binary word either has a factor in $F=\{000,111,0101,1010,0110110,1001001\}$ or contains $(0011)^{4}$ as a factor. Moreover, for any factor $f[1, d]$ in $F$, the factor $f_{d} f_{d-1} \ldots f_{2} f_{1} f_{2} \ldots f_{d-1} f_{d}$ is a repetition of exponent at least $\frac{7}{2}$.

Case $k=3$ :

Let $F$ be the set of words obtained by permutations of the letter alphabet of the words in $\{00,0101,0121012,012012012\}$. A computer check shows that every ternary word $w$ of length 58 contains a factor $w[b, e]$ such that the factor $w_{e} w_{e-1} \ldots w_{b+1} w_{b} w_{b+1} \ldots w_{e-1} w_{e}$ contains a cube.

The following word of length 57 does not contain such a factor:

010201210212012021021012012010210210120120210212012102010.

Case $k \geq 4$ :

It is clear that any level-coloring of the complete binary tree of height 1 contains a repetition of exponent at least $\frac{3}{2}$.

Acknowledgements. We would like to thank an anonymous referee whose comments helped us improve the readability of the paper.

\section{REFERENCES}

[1] A. Aberkane and J. Currie, There exist binary circular 5/2+ power free words of every length. Electron. J. Comb. 11 (2004) R10.

[2] J. Chalopin and P. Ochem, Dejean's conjecture and letter frequency. RAIRO-Theor. Inf. Appl. 42 (2008) 477-480.

[3] F. Dejean, Sur un théorème de Thue. J. Combin. Theory. Ser. A 13 (1972) 90-99.

[4] J. Grytczuk, Nonrepetitive colorings of graphs - a survey. Int. J. Math. Math. Sci. (2007) doi: $10.1155 / 2007 / 74639$.

[5] P. Ochem, A generator of morphisms for infinite words. RAIRO-Theor. Inf. Appl. 40 (2006) 427-441.

[6] A. Pezarski and M. Zmarz, Non-repetitive 3-Coloring of subdivided graphs. Electron. J. Comb. 16 (2009) N15.

Communicated by G. Richomme.

Received November 2, 2010. Accepted August 5, 2011. 